\title{
Lettere al signor professore Wilcken.
}

\section{XLVI.}

Roma, 21 Maggio 1908.

Pregiato Amico,

Nella dissertazione del Dedel, Historia critica bibliothecae Alexandrinae, 1823, pag. 33, trovo «arabice et latine», un passo di Hadj Khalifa (ex cod. ms. d' Hossonii), di cui la traduzione suona così: «Ferunt ibn Abbasum imprimis improbare scribendi artem ... Cum autem quodam tempore vir aliquis ad eum venisset, dicens se librum scripsisse, quem ipsi offerre cuperet eumque revera detulisset, ibn Abbasus scripturam aqua delevit, et interrogatus cur ita faceret, hac de causa inquit, quod homines, si libros scribant, illis quae scripto consignaverint, confisi, memoriam non amplius exercebunt.»

È orvia qui, ed, a mio credere, tutt' altro che inopportuna ed inutile, una osservazione che mi stupisco non sia stata fatta dal Dedel, cioè che, con altri personaggi e con qualche variante, abbiamo in questo racconto arabo un sicuro riscontro al $\mu \tilde{v} \vartheta o_{s}$ che si legge in Platone

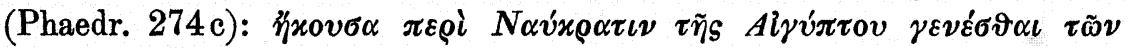

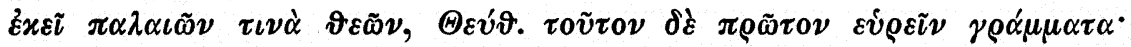

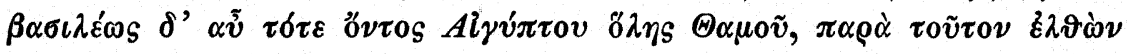

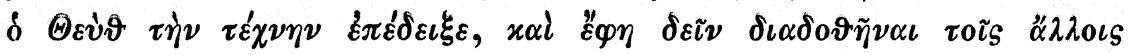

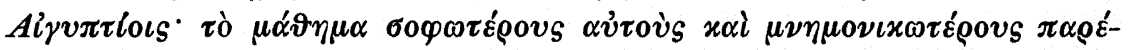

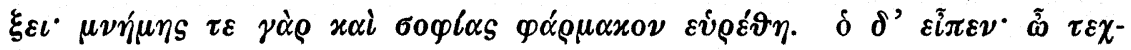

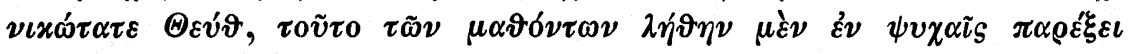

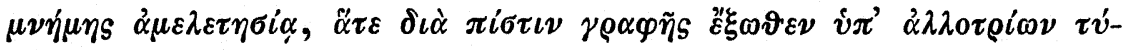

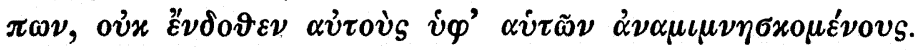

Tramite probabile Alessandria: giacchè non mancano esempî di racconti alessandrini de' quali troviamo un' eco in racconti arabi (cf. Crusius, Comm. ad libell. de prov. alexandr. 1895, p. 35; Carra de Vaux, Les mille et une nuits, in Revue des deux mondes, 1906, p. 154).

Aff ${ }^{m o}$. Suo

Giacomo Lumbroso. 


\section{XLVII.}

Roma, 22 Maggio 1908.

Pregiato Amico,

Più e più volte, considerandone la singolarità e fiutandone la non comune portata, io mi sono chiesto quale abbia potuto essere il vero senso della frase di Dione Crisostomo nella sua Orazione agli Alessan-

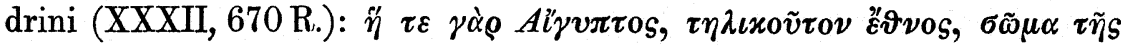

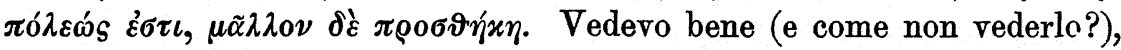
che si trattava di un doppio aspetto dell' $A$ '̈ $\gamma v \pi \tau$ os in correlazione alla $\pi \delta \lambda_{\iota s}$, e per conseguenza, di due pregi della $\pi$ ó $\iota_{s}$, de' quali il secondo doveva essere più ragguardevole del primo. Ma poi, in cerca del senso esatto e preciso da attribuirsi qui alla parola $\sigma \tilde{\omega} \mu \alpha$ ed alla parola $\pi \varrho 0 \sigma \vartheta \eta x \varkappa \eta$, le due chiavi del senso storico della frase, io mi smarrivo, ed ora rinunziavo a fantasticare ed arzigogolare, tra me e me, intorno ad essa, ed ora tornavo a pensarci su. Il fatto è che soltanto dä poco tempo sento di potermi fermare ad una interpretazione alquanto probabile e giustificabile.

La $\pi \delta \lambda_{\iota S}$ è (se posso far così parlare Dione) $\pi \varrho o \alpha \nu l \sigma \chi o v \sigma \alpha \tau \tilde{\eta} S$

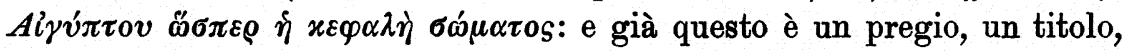

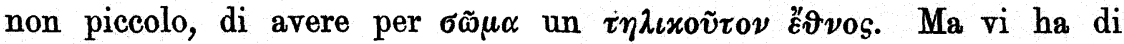
più e di meglio, s'affretta ad aggiungere l'oratore. L' $A l^{\prime} \gamma v \pi \tau$ os è piuttosto una $\pi \varrho 0 \sigma \vartheta \eta^{\prime} \varkappa \eta$ della $\pi$ ódıs. La $\pi \delta \lambda_{\imath c}$ stessa è dunque un tutto, uno stato, un regno a sè. Indipendentemente dall' essere la capitale

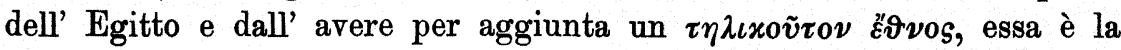

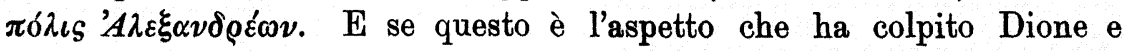
che egli ha voluto accennare, certo nessun testo meglio del suo può darci un' idea del posto che tenne Alessandria nel mondo antico.

Suo

Giacomo Lumbroso.

\section{XLVIII.}

\section{Roma, 23 Maggio, 1908.}

Mio pregiato amico,

Nella spiritosa lettera di Adriano, o degna di Adriano, concernente Alessandria (Vopisc., Saturnin. 8), «civitas in qua nemo vivat otiosus. alii vitrum conflant, aliis charta conficitur, alii linifiones, omnes certe cuiuscumque artis et videntur et habentur. podagrosi quod agant habent, habent caeci quod faciant, ne chiragrici quidem aput eos otiosi vivunt», non si prenda la pennellata per una caricatura. Tale a un di 
presso è il ritratto della Cina d' or fa tre secoli (Botero, Relationi universali, 1605 , I p. 125 , II p. 63): "gli oziosi nô si comportano in alcuna maniera. Ogni uno hà da fare secondo le sue forze: chi fa qualche cosa con la mano; chi co'l piede, chi cô l'occhio, chi con la lingua» ecc. Tale il ritratto dell' Amsterdam dei tempi di Voltaire (Correspond., ed. Thomine, I, p. 24): «de cinq cent mille hommes qui habitent Amsterdam, il n'y en a pas un d' oisif... On ne connaît que le travail.» Tale il ritratto di Nuova-York ai giorni nostri (Bourget, Reconnaissance p. 40): «Pour vivre à New-York il faut travailler. Tout le monde y fait quelque chose. Il n' y a pas de place pour un oisif.»

Fin dal primo suo sviluppo, meravigliosamente rapido (Ammian. XXII, 16, 15), la Nuova-Ellade d' oltre mare dovette fare alla VechiaEllade ateniese (Lucian., Navigium) la stessa impressione che il Nuovo Mondo alla vecchia Europa.

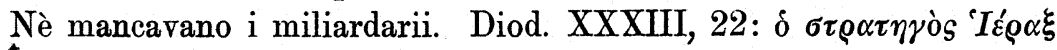

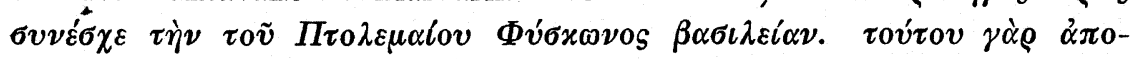

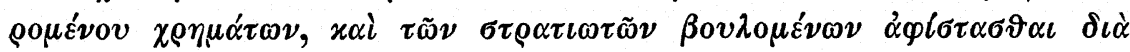

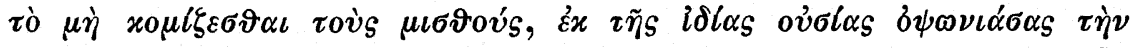

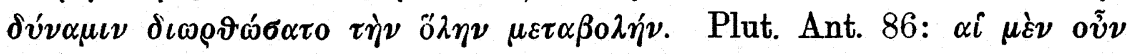

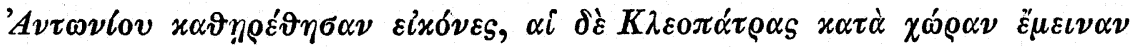

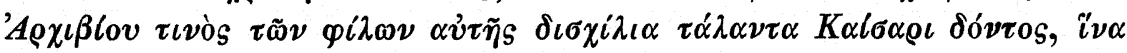

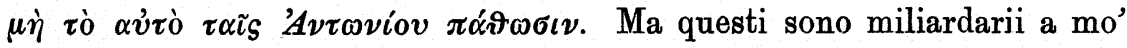
dei Richelieu e dei Mazzarini, quasi soci dei Re. Dico che non mancavano i miliardarii privati. Iustin. XXXIX, 1, 4: «Ptolemaeus rex Aegypti bello a Demetrio petitus, inmittit iuvenem quendam Aegyptium, Protarchi negotiatoris filium, qui regnum Syriae armis peteret.» Ios. antiqq.

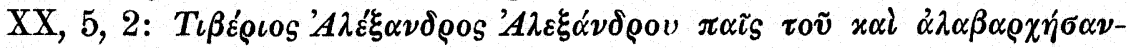

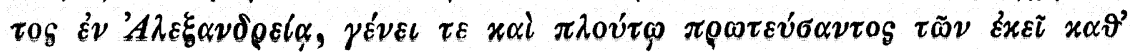

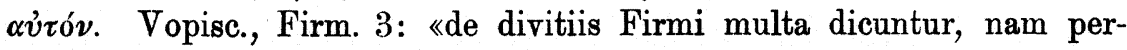
hibetur tantum habuisse de chartis ut publice saepe diceret, exercitum se alere posse papyro et glutine.»

«Unus illis deus nummus est», dice Adriano, o chi per lui, in quella lettera: altro che l' $E \tilde{i}_{S} Z_{\varepsilon} \dot{v} S \delta$ $\delta \alpha \rho_{\varrho} \alpha \pi \iota_{S}$ delle loro labbra!

Suo

Giacomo Lumbroso.

XLIX.

Pregiato amico,

Roma, 24 maggio, '908.

Trattando dei progressi che fece in Alessandria la Chirurgia, il Parthey (Alexandrinische Museum p. 177) immagina quelli che vi do- 
vette pur fare l' Arte di bendare con fascie le ferite, al contatto dell' antichissima pratica indigena di bendare con fascie le mummie: «Bei dem zweckmässigen Anlegen des Verbandes benutzten die Alexandriner ohne Zweifel die tausendjährige Erfahrung der Ägypter ...».

Questo mi fa pensare ad un patto tra Entafiasta e Medico, che figura in un lepido epigramma dell' Antologia Palatina (XI, 125):

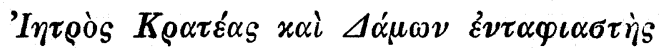

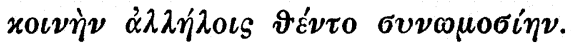

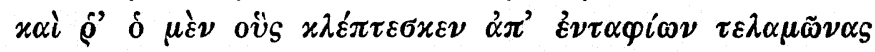

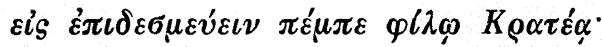

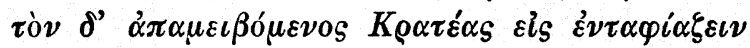

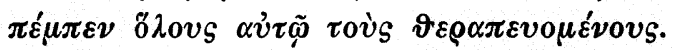

In $\tau \varepsilon \lambda \alpha \mu \tilde{\omega} \nu \alpha_{S}$ ritroviamo quel nome delle «fascie» che è in Erodoto VII, 181 dove si vedono i Persiani medicare con cura le ferite di certo

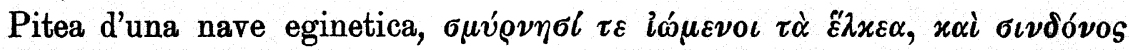

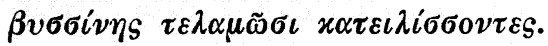

Suo

Giacomo Lumbroso.

L.

Pregiato Amico,

Roma, 25 Maggio 1908.

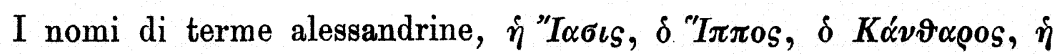

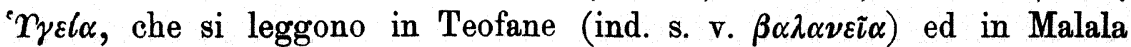
(ed. Bonn. p. 192), credo che meritino di essere notati come indizi di opere d'arte, Così dicendo, io mi appoggio sopra tutto ad una osservazione del Reiske (ad Const. Porph. II p. 143): «mos illis temporibus thermas publicas a statuis ibi erectis nominandi. ita apud Constantinopolim ' $A \chi \iota \lambda \lambda_{\varepsilon} \dot{v}_{S}$ erat nomen sic dictarum a celebri statua Achillis ibi conspicua. ita apud Tripolim erat balneum $\delta$ "Ixooos dictum a statua Icari ibi posita.» Quelle appellazioni alessandrine equivalgono, dunque, a notizie, magre sì, ma notizie, di statue od altre opere artistiche. La

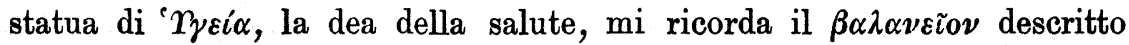

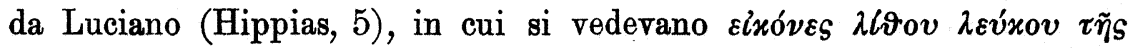

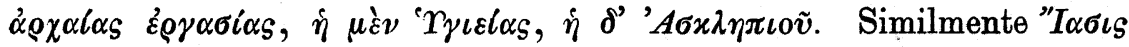
sarà nome e statua della ninfa di cui abbiamo notizia in Pausania $(6,22,7)$. E la supposizione di queste due immagini pagane, riceve luce altresì da un episodio di Alessandria cristiana, che trovasi in un testo siriaco così tradotto recentemente nella Patrologia Orientale del 
Didot (II, 1, p. 33): «Le peuple s'enflamme, apporte toutes les idoles des dieux des païens, soit qu' elles se trouvent dans les bains $(\beta \alpha \lambda \alpha-$ $\nu \varepsilon \tilde{\alpha} \alpha)$ ou dans les maisons, les place en tas et y met le feu.» Anche il nome $K \alpha \dot{v} \vartheta \boldsymbol{\alpha}$ os $_{\text {è }}$ un documento d'arte: cf. Mirabilia urbis Romae c. 20, 2: «in paradiso s. Petri est cantarum columpnis porphireticis ornatum; que tabulis marmoreis cum griphonibus conexe, precioso celo ereo cooperte, cum floribus et delfinis ereis et deauratis aquas fundentibus»; S. Paulin. Nol. epist. ad Pammachium, p. 73 ed. Veron.: «cantharum ministra manibus et oribus nostris fluenta ructantem, fastigatus solido aere tholus ornat et inumbrat, non sine mystica specie, quattuor columnis salientes aquas ambiens» (presso Huelsen, in Bull. dell' Inst. archeol. germanico, sezione romana, vol. XVIII, 1903, p. 41 e segg.).

Suo deditiss:

Giacomo Lumbroso.

LI.

Roma, 28 Maggio, '908.

Mio pregiato e caro amico,

È gran tempo che ho in animo di sottopor Le una osservazione concernente lo studio delle cose alessandrine.

Quando l' Egitto cadde in potere dei Macedoni e si popolò di

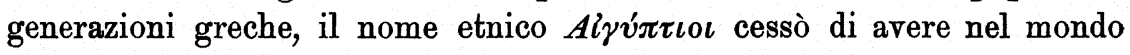
un unico significato. Rimase bensì quel nome a dinotare, occorrendo, gli Egiziani propriamente detti, ma dinotò anche, via via, non solo gli

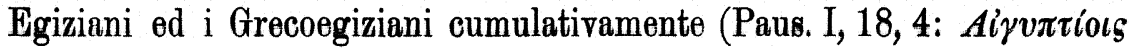

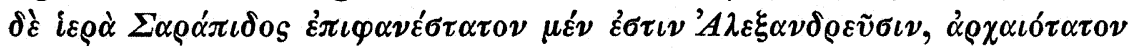
$\delta \dot{\varepsilon} \varepsilon \dot{\varepsilon}\left(M \varepsilon^{\prime} \mu \varphi \varepsilon \iota\right)$, ma molte volte, poichè questo era l' elemento civilmente e politicamente più in vista, i soli Grecoegiziani, e soprattutto, data la importanza della $\pi$ ódıs, gli Alessandrini.

Polibio XXVII, 8 raccontando la gara tra l' atleta Aristonico, man-

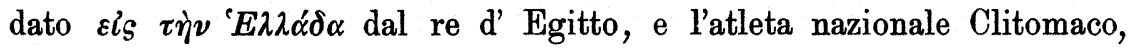

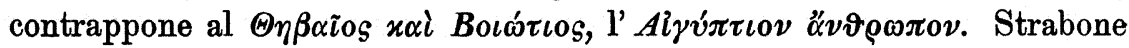
XII, 558, XVII, 796 parlando della fuga di Tolemeo Aulete, che in altri scrittori comparisce come "seditione flagitatus Alexandreae», come cacciato dai «clubs» alessandrini (Trog., Prol. XL: Dio Chrys. or. XXXII),

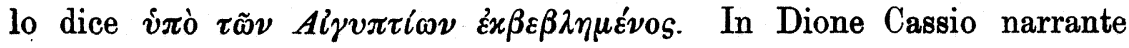

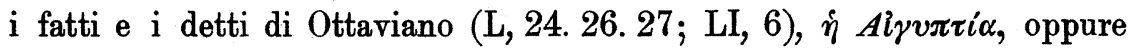

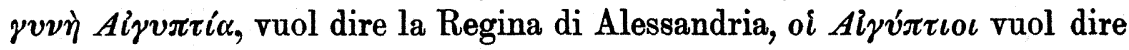


l' Esercito alessandrino. In Plutarco, quando Marc' Antonio celebra in quella città il suo trionfo armeno, è $\tau \grave{\alpha} \quad x \alpha \lambda \grave{\alpha} x \alpha i \quad \sigma \varepsilon \mu \nu \grave{\alpha} \quad \tau \tilde{\eta}_{S} \pi \alpha \tau \rho i \delta o s$

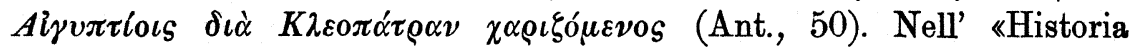
Augusta», si scriverà: «cogitabat Aurelianus ne, si perturbidam civitatem vidisset Saturninus, quo eum natura ducebat, eo societate quoque hominum duceretur. et mente quidem divina. nam ut primum Aegyptii magnam potestatem ad se venire viderunt, statim etc.» (Vopisc., Saturn.7).

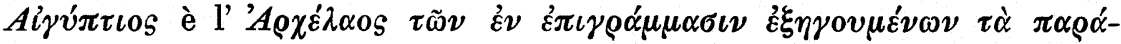

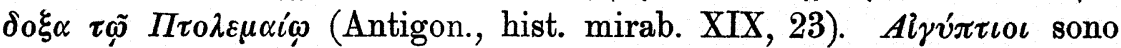
gli uditori accorsi ad una celebre scuola di Smirne (Philostr., Vit.

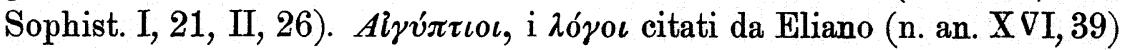
a proposito di animali rari esposti in Alessandria. Kovøó $\eta \mathbf{s} A i \gamma v$ $\pi \tau \ell \omega \nu$ in Gregorio Nazianzeno (1611, t. 2. p. 12; Migne, t. 3. p. 1069)

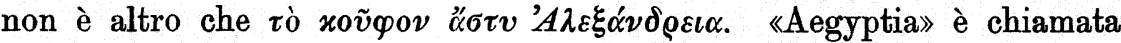
la «gens libris exarandis et compingendis solertior caeteris», in lettera dell' imp. Costante ad Athanasio (Op. om. 1698, t. I p. XXXIII). Ecc.

Quindi i molti esempi evidenti potendo gettar luce su quelli a cui l'evidenza manca, c'è tutto un materiale da vagliare in questo senso e da avocare per così dire ad Alessandria.

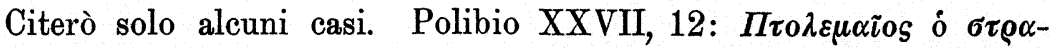

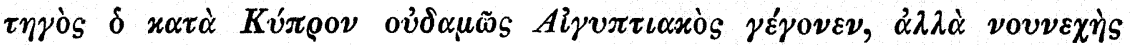

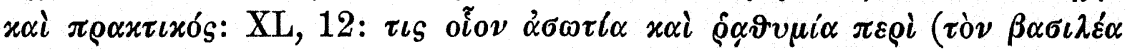

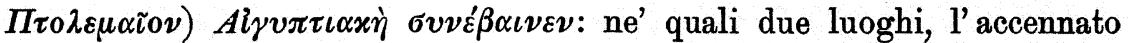
uso ci aiuta ad affermare con sufficente sicurezza che quell' $A i \gamma v \pi \tau \iota \alpha-$

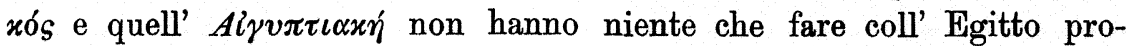
priamente detto, e si riferiscono unicamente all Amministrazione ed alla Corte alessandrina del tempo di Tolemeo VI. Ammiano XXVIII, 5, 14: «apud Burgundios removetur rex si sub eo fortuna titubaverit belli vel segetum copiam negaverit terra, ut solent Aegyptii casus eius modi suis adsignare rectoribus»: dove in grazia di quello stesso uso possiamo con maggiore franchezza addossare agli Alessandrini il «fortuita ad culpam trahere» di Tacito (ann. IV, 64). Petron. Satyr. 34: «Pictura quoque non alium exitum fecit, postquam Aegyptiorum audacia tam magnae artis compendiariam invenit»: dove dunque, risparmiando la pittura egiziana, si potrà più facilmente ravvisare coll' Helbig (Campan. Wandmalerei) un' audacia alessandrina.

Cordialmente Suo

Giacomo Lumbroso. 
LII.

Roma, 31 Maggio 1907.

Pregiato amico,

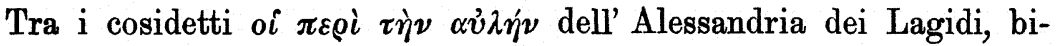

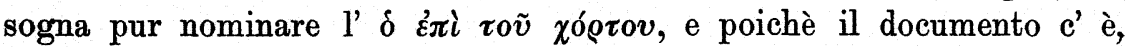
bisogna pur documentare l' esistenza di questo ufficio. Per fortuna il documento è così grazioso che se invece di essere a stampa in Ateneo (XIII, 583 a), uscisse adesso adesso dopo duemil' anni, anche solo super-

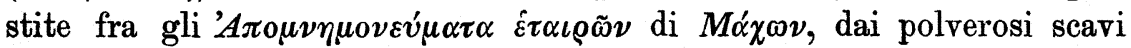
dell' Arsinoite o dell' Ossirinchite, indubbiamente per mesi e mesi farebbe la gioia dei filologi di tutta Europa e sarebbe tradotto in prosa od in rima in tutte le lingue:

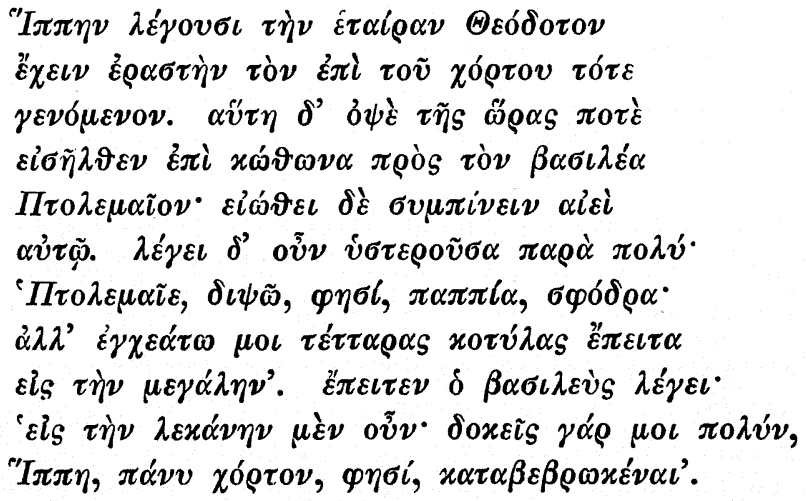

Per non uscire dal tema $\tau \tilde{\omega} \nu \boldsymbol{\pi} \varepsilon \varrho i \tau \eta \dot{\nu} \alpha \hat{v} \lambda \dot{\eta} \nu$, noto soltanto in aggiunta

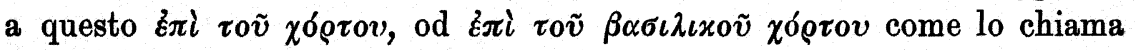
Eustazio (ad Iliad. $\varphi^{\prime}$, p. 1307, l. 33), i termini $\chi \varrho \rho \tau 0 \vartheta \eta \dot{x} \eta$ (Wilcken,

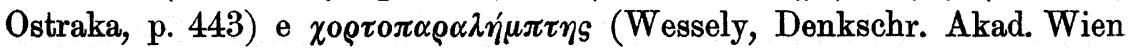
XXXVII, 2, pp. 102. 239) che son venuti fuori nei Papiri.

Affez ${ }^{\mathrm{m}}$.

Giocomo Lumbroso.

\section{LIII.}

Roma, 1 Giugno, '908.

Pregiato Amico,

Guadagnarsi il pane, procacciarsi un lucro, dicesi in greco $\pi 00$ i$\xi \varepsilon \imath \nu, \pi 0 \rho \iota \sigma \mu \delta s$; colla quale espressione si abbraccia ogni sorta di $\varepsilon^{\circ} \rho \gamma \alpha$, di $\tau \varepsilon^{\prime} \chi \nu \alpha \iota$, di $\dot{\varepsilon} \pi \iota \tau \eta \delta \varepsilon v ́ \mu \alpha \tau \alpha$, conducenti al sostentamento od al lucro. 
Ma oltre ai tanti esempî in questo senso, che si incontrano anche in Filone e dei quali ha fatto un diligente spoglio il Conybeare nel suo dottissimo Commento alla Vita Contemplativa (Oxford, 1895, p. 103-104), e' è un passo dello scrittore alessandrino, in cui $\pi 0 \varrho i$ -

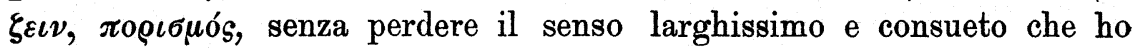
detto, par che sia venuto ad assumere, in Alessandria, anche un senso

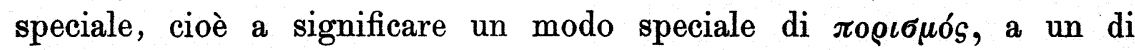
presso come la parola é o lavoro, trovasi in Aristea ( $\$ 108$, Wendland) ed in qualche Papiro,

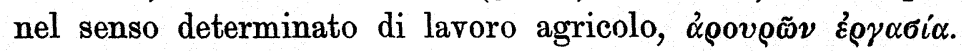

Il passo di Filone è quello in cui racconta certe sommosse antisemitiche alessandrine, ed espone, l'una immediatamente accanto all' altra, le due conseguenze che quelle sommosse ebbero pei Giudei d'ogni

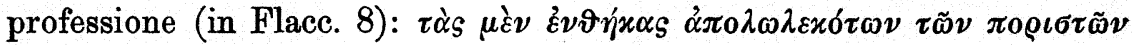

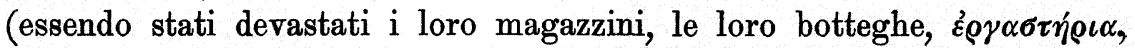

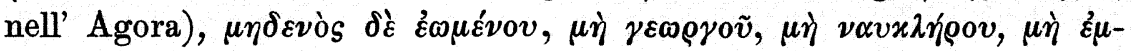

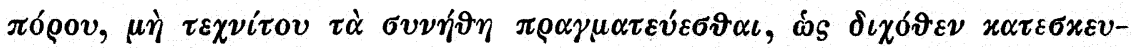

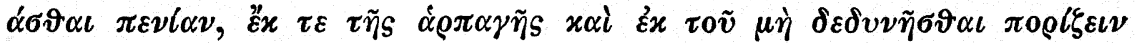

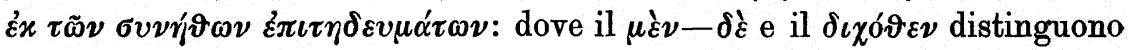
i $\pi \circ \varrho \iota \tau \tau \alpha \ell$ propriamente detti da tutti gli altri professionisti, quantun-

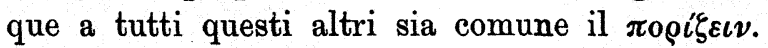

Suo

Giacomo Lumbroso.

LIV.

Viareggio, 11 Giugno '908.

Pregiato Amico,

Leggendo il Diario romano (1485-1524) di Sebastiano Tedallini, testè pubblicato nella nuova raccolta dei «Rerum italicarum scriptores» (t. XXIII, $\mathrm{p}^{\text {te }}$ III), mi sono imbattuto in questa notizia (p. 305): «A dì 25 de agosto 1502, come sallì uno garzone de Antonino Freiapane, che si chiamava Sebastiano, in colonna Antonina, dallo canto de fora con le mano e colli piedi, senza altra cosa, et fu lo dì de Santo Bartolomeo: dicono molti vecchi che non ce sallì mai nisciuno». E la notizia mi ha ricordato quest' altra che è nel «Recueil des inscriptions grecques et latines de l'Egypte» del Letronne (II, p. 488): «Pour preuve de ce que peut une extrême agilité jointe à l' habitude que donne un long exercice, M. Hector Horeau, auteur du Panorama d'Egypte et de Nubie, m' a dit avoir vu un jeune Arabe monter jusqu' au sommet de l'obélisque resté à Louqsor, en mettant ses pieds et ses mains dans 
le creux des hiéroglyphes de la bande du milieu, qui sont, comme on sait, profondément entaillés.»

Ora queste due notizie mi trarrebbero a ravvisare, non dico un documento, ma un indizio, di simili agilità ed arditezze, in una medaglia alessandrina dei tempi imperiali, descritta dallo Zoega, Numi Aeg., p. 268, no 39: «Nilus decumbit dextra tenens cornucopiae, cuius summitati insidet puer, adsidente altero ad imum cornu, sinistra vero arundinem cum circumludentibus pueris quatuor: e regione erectus est obeliscus, quem velut ascensurus ulnis amplectitur septimus puer basi insistens, octavo ibidem ad imam obelisci basin adstante.»

Piccolezza, senza dubbio. Ma nè io la dò, nè Lei la piglia, per più di quel che vale, cioè di un' ipotesi di straordinarie bravure e popolari scenette alessandrine di questo genere.

Suo

Giacomo Lumbroso.

LV.

Viareggio, 14 Giugno, '908.

Pregiato Amico,

Il detto di Tolemeo $\mathrm{V}$ sui pílo mentre si accingeva ad una guerra contro Seleuco IV re di Siria, non è, come parrebbe dalle moderne storie dei Lagidi (Sharpe, I, 253; Mahaffy, 313; Bouché-Leclercq, I, 399) unicamente riferito da San Girolamo. Lo si trova già in

Diod. XXIX, 29 [Exc. Vat.]: ö $\tau \iota$

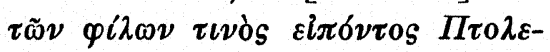

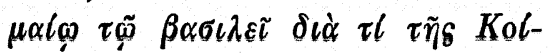

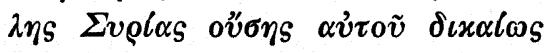

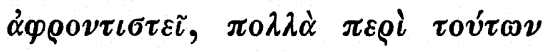

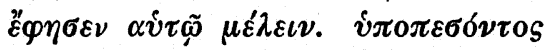

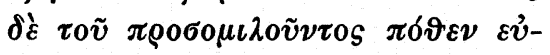

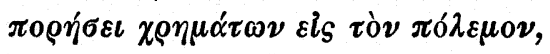

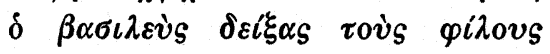

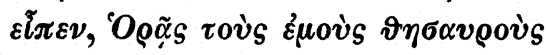
$\pi \varepsilon \varrho \iota \pi \alpha \tau o \tilde{v} \nu \tau \alpha_{S} ;$
[Hieron. in Dan. XI, 20, p. $711 \mathrm{~A}$ : «Porro Porphyrius non vult hune esse Seleucum, sed Ptolemaeum Epiphanem, qui Seleuco sit molitus insidias, et adversum eum exercitum praepararit, et idcirco veneno sit interfectus a ducibus suis. quod cum unus ab illo quaereret, tantas res moliens ubi haberet pecuniam: respondit sibi amicos esse divitias. quod quum divulgatum esset in populis, timuerunt duces ne auferret eorum substantiam, et idcirco eum maleficis artibus occiderunt».]

Polibio (XXV, 7), come osserva M. Bouché-Leclereq, e Diodoro, come si vede, non accennano («autant du moins qu'on en peut juger 
par leurs fragments»), alla morte violenta di cui parla Porfirio. Stando così le cose, non possiamo cavar altro da Diodoro se non una testimonianza intorno alla grande ricchezza, d'origine regia, dei $\varphi \iota \lambda_{o \iota}$ (Plut.

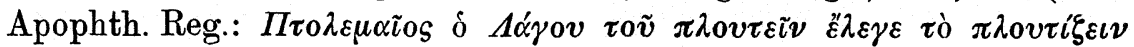
siv $\alpha \iota \beta \alpha \sigma \iota \lambda \iota x \omega ́ \tau \varepsilon \varrho o \nu)$, ed alla possibilità che quella grande ricchezza venisse in aiuto al Principe nelle circostanze straordinarie ed urgenti (cf. Diod. XXXIII, 22), salvo i casi in cui le fazioni, le turbolenze, le ostilità, gli dessero occasione di far denari coi supplizi e colle confische (Dio Cass. LI, 5).

Suo

\section{Giacomo Lumbroso.}

\section{LVI.}

Viareggio, 22. Giugno, 1907.

Pregiato Amico,

In tutta l' invettiva di Filone contro Flacco, in tutto l' episodio

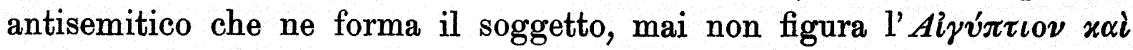

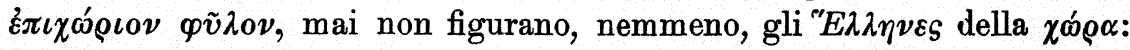
figurano soltanto gli ' $A \lambda \varepsilon \xi \alpha \nu \delta \rho \varepsilon \tilde{u} s$. Sono espressamente ed esclusivamente gli ' $A \lambda \varepsilon \xi \alpha \nu \delta \varrho \varepsilon \tilde{L}$, quelli di cui parla Filone, quelli che chiedono al Prefetto, in cambio del loro appoggio e favore, l' atteggiamento ostile verso i Giudei. Quando dunque, in un passo dell' invettiva, Filone rampogna Flacco per essersi lasciato imporre quel programma,

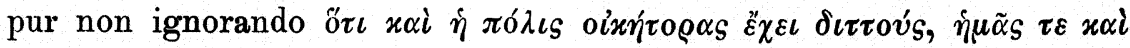

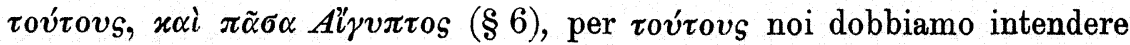
gli ' $A \lambda \varepsilon \xi \alpha \nu \delta \varrho \varepsilon \check{s}$, e quando, a giustificare in certo modo la libertà ch' ei

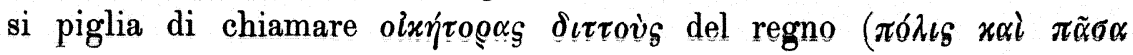
$\left.A i^{\prime} \gamma v \pi \tau o s\right)$ i Giudei e gli Alessandrini, Filone soggiunge tosto ö $\tau \iota$ ov่x

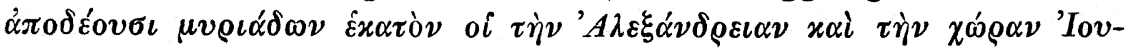

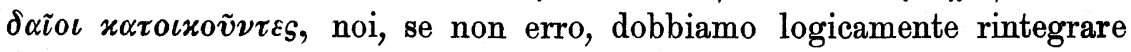
il parallelo e leggere tra le righe un numero approssimativamente uguale, un numero non guari diverso, di $\mu v \varrho \alpha_{\alpha} \delta \varepsilon_{S}$ componenti nel primo secolo dell' impero il $\pi \lambda \tilde{\eta} \vartheta o_{S}$ degli ' $A \lambda \varepsilon \xi \alpha \nu \delta \rho \varepsilon \tilde{\varepsilon} s$, tra permanenti nella $\pi$ ód cs (eran già 300000 negli ultimi tempi dei Lagidi: Diod. XVII, 52) e soggiornanti nella $\chi \omega ́ \varrho \alpha$, dove si offrivano ad essi tante imprese commerciali, industriali ed agricole (Wilcken, Ostraka, I, 415).

Dico «se non erro»; e Le stringo cordialmente la mano.

Suo

Giacomo Lumbroso. 


\section{LVII.}

Viareggio, 25 Giugno 1908.

\section{Pregiato Amico,}

Non è l' unico aiuto che si abbia a fissare la cronologia di certi eventi del regno di Tolemeo IV, ma è pur da prendersi in considera-

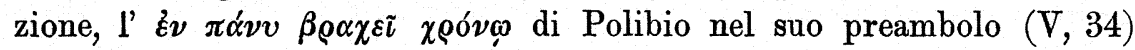

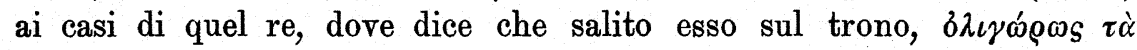

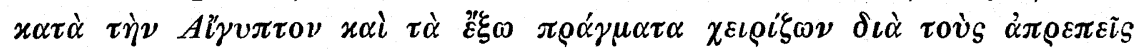

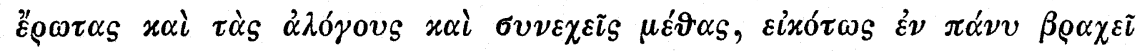

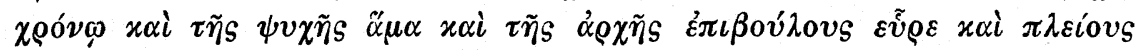
(stando alle frammentarie particolarità di cui abbiamo notizia: $1^{\circ}$ Cleomene colla sua congiura, $\mathrm{V}, 34 ; 2^{\circ}$ Teodoto col suo attentato, $\mathrm{V}, 81$; $3^{\circ} \mathrm{gl}$ ' Indigeni colla loro ribellione, $\left.\mathrm{V}, 107\right)$.

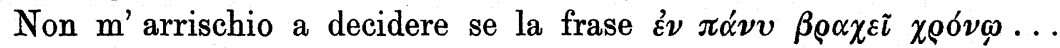

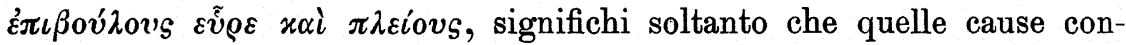
dussero prestissimo a quegli effetti, oppure significhi altresì che quegli effetti si produssero a pochissima distanza l'un dall' altro, ma qualunque sia l'interpretazione migliore, sempre la frase in sè mi par che meriti di essere notata come la prima delle basi fornite da Polibio alla cronologia di cui si tratta.

Aff ${ }^{\text {to }}$ Suo

Giacomo Lumbroso. 